\title{
Naturalness of Nonlinear Scalar Self-Couplings in a Relativistic Mean Field Theory for Neutron Stars
}

\author{
Cláudio Maekawa*, Moisés Razeira ${ }^{\dagger}$, César A.Z. Vasconcellos ${ }^{\dagger}$, Manfred \\ Dillig** and Bardo E.J. Bodmann* \\ *Departamento de Física, Fundação Universidade Federal de Rio Grande, CEP 96201-900 Rio \\ Grande, Brazil \\ ${ }^{\dagger}$ Instituto de Física, Universidade Federal do Rio Grande do Sul, CEP 91501-970 Porto Alegre, \\ Rio Grande do Sul, Brazil \\ **Institut für Theoretische Physik III, der Universität Erlangen-Nürnberg, D91058 Erlangen, \\ Germany \\ ${ }^{\ddagger}$ Instituto de Física e Matemática, Universidade Federal de Pelotas, CEP 96010-900 Pelotas, Rio \\ Grande do Sul, Brazil
}

\begin{abstract}
We investigate the role of naturalness in effective field theory. We focus on dense hadronic matter using a generalized relativistic multi-baryon lagrangian density mean field approach which contains nonlinear self-couplings of the $\sigma, \delta$ meson fields and the fundamental baryon octet. We adjust the model parameters to describe bulk static properties of ordinary nuclear matter. Then, we show that our approach represents a natural modelling of nuclear matter under the extreme conditions of density as the ones found in the interior of neutron stars.
\end{abstract}

\section{NATURALNESS AND EFFECTIVE MODEL}

In the building up of an effective model for the nuclear many-body problem, a natural way to classify their contributions is to expand the lagrangian density in terms of the characteristic scales of QCD[1]. The restriction of the description to a limited physical domain reveals the relevant physical phenomena are in general dominated by a few specific long-range degrees of freedom, while the dynamics at shorter length scales, which corresponds to heavier physical degrees of freedom, may be integrated out and are implicitly absorbed in the various coupling parameters of the theory. Expanding the lagrangian density of the effective model order by order, we assume the unknown dimensionless coupling parameters of the theory are natural, i. e. of the order of unity. The naturalness assumption makes it possible to formally sum up in a compact form, any expansion of the lagrangian density to any desired order.

To establish naturalness we follow the ideas of modern effective field theory (EFT) and apply the naive dimensional analysis (NDA) proposed by Manohar and Georgi[1]. To set the scale and expansion parameters we rely on the fact that the mean scalar $\sigma$ and vector $\omega$ potentials may exhaust the overwhelming part of the effective $\mathrm{NN}$ interaction and their expected values at saturation can be considered small when compared to the bare nucleon mass $M$, allowing to use the ratios $\sigma / M$ and $\omega / M$ explicitly as expansion 
parameters.

The interaction lagrangian which involves the isoscalar-scalar meson field $\sigma$ and the isoscalar-vector meson field $\omega$ coupled to the nucleon field is then defined in effective theory as [1]

$$
\begin{aligned}
L_{e f f} & =\sum_{i, k} \frac{c_{i, k}}{i ! k !}\left(\frac{\sigma}{f_{\pi}}\right)^{i}\left(\frac{\omega}{f_{\pi}}\right)^{k}\left(\frac{\partial \text { or }_{\pi}}{M}\right)\left(\frac{\bar{\psi} \Gamma \psi}{f_{\pi}^{2} M}\right)^{\ell} f_{\pi}^{2} \Lambda^{2} \\
& \rightarrow \sum_{i, k} \frac{\tilde{c}_{i, k}}{i ! k !}\left(\frac{g_{\sigma} \sigma}{M}\right)^{i}\left(\frac{g_{\omega} \omega}{M}\right)^{k}\left(\frac{\partial o r m_{\pi}}{M}\right)\left(\frac{\bar{\psi} \Gamma \psi}{f_{\pi}^{2} M}\right)^{\ell} f_{\pi}^{2} \Lambda^{2}
\end{aligned}
$$

where, in the second expression, we have used to eliminate the $f_{\pi}$ factor: a) the Goldberger-Treiman[2] relation $\left(g_{\sigma} f_{\pi} \sim M\right)$; b) the Kawarabayashi-Suzuki-RiazuddinFayyazuddin[3] relation $\left(m_{\omega}^{* 2}=2 g^{2} f_{\pi}^{* 2}\right)$ with $g$ denoting the hidden-gauge coupling constant and $f_{\pi}^{*} \sim f_{\pi}$ the pion decay constant for nuclear matter; c) $m_{\omega}^{* 2}=2 g^{2} f_{\pi}^{* 2}$; $g / 2=g_{\omega} / 3$ (See [4]); d) and finally $\left|m_{\omega}^{*}\right|_{\rho_{0}}=\left|m_{\omega}-M+M^{*}\right|_{\rho_{0}} \sim M / 2$. These expressions contain unknown expansion coefficients (overall coupling constants) $\tilde{c}_{i, k}$; a direct generalization of this expression may involve additional fields such as the $\pi, \rho, \delta$ meson fields and the photon, as well as, factorial counting factors, since the NDA rules presumably describe scattering amplitudes at the tree level. In the expression above $\psi$ represents a baryon field, $\Gamma$ is a Dirac matrix and derivatives are denoted by $\partial$. The overall dimensionless coefficients, after the dimensional factors and appropriate counting factors are extracted, are of order $\mathscr{O}(1)$ if naturalness holds.

In the natural limit our effective approach above gives rise to a nonlinear exponential coupling (exponential model)

$$
L_{e f f}\left(c_{i, k}=1\right) \longrightarrow \exp \left(\frac{g_{\sigma} \sigma}{M}\right) \exp \left(\frac{g_{\omega} \omega}{M}\right)\left(\frac{\partial o r m_{\pi}}{M}\right)\left(\frac{\bar{\psi} \Gamma \psi}{f_{\pi}^{2} M}\right)^{\ell} f_{\pi}^{2} \Lambda^{2} .
$$

For practical applications, with a full QCD calculation presently beyond any reach, we consider the natural limit of the extended effective lagrangian model which contains additionally nonlinear self-couplings of the $\rho, \delta$ meson fields interacting with the fundamental baryon octet

$$
\begin{aligned}
\mathscr{L} & =\sum_{B} \bar{\psi}_{B}\left\{\gamma_{\mu}\left(i \partial^{\mu}-g_{\omega B}^{\star} \omega^{\mu}-\frac{1}{2} g_{\rho B}^{\star} \tau_{B} \cdot \rho^{\mu}\right)-\left(1-\frac{g_{\sigma} \sigma}{M_{B}}\right)^{\eta} m_{\alpha B}^{\star} M_{B}\right\} \psi_{B} \\
& +\frac{1}{2}\left(\partial_{\mu} \sigma \partial^{\mu} \sigma-m_{\sigma}^{2} \sigma^{2}\right)+\frac{1}{2}\left(\partial_{\mu} \vec{\delta} \cdot \partial^{\mu} \vec{\delta}-m_{\delta}^{2} \vec{\delta}^{2}\right)+\frac{1}{2}\left(m_{\omega}^{2} \omega_{\mu} \omega^{\mu}-\frac{\omega_{\mu v}}{2} \omega^{\mu v}\right) \\
& +\left(\frac{1}{2} m_{\rho}^{2} \rho_{\mu} \cdot \rho^{\mu}-\frac{1}{4} \rho_{\mu v} \cdot \rho^{\mu v}\right)+\sum_{\lambda} \bar{\psi}_{\lambda}\left(i \gamma_{\mu} \partial^{\mu}-m_{\lambda}\right) \psi_{\lambda}
\end{aligned}
$$

where $\omega_{\mu v}=\partial_{\mu} \omega_{v}-\partial_{v} \omega_{\mu}$ and $\rho_{\mu v}=\partial_{\mu} \rho_{v}-\partial_{v} \rho_{\mu}$, with $g_{\alpha \sigma B}^{\star} \equiv m_{\alpha B}^{\star} g_{\sigma} ; g_{\beta \omega B}^{\star} \equiv$ $m_{\beta B}^{\star} g_{\omega} ; g_{\gamma \rho B}^{\star} \equiv m_{\gamma B}^{\star} g_{\rho} ; g_{\alpha \delta B}^{\star}=m_{\alpha B}^{\star} g_{\delta} ; m_{\kappa B}^{\star} \equiv\left(1+\left(g_{\sigma} \sigma+g_{\delta} \tau_{3 B} \delta_{3}\right) / \kappa M\right)^{-\kappa}$, and with $(\kappa=\alpha, \beta, \gamma)$.

Then, we confront the predictions of our approach for the following choices of the parameters ( $\alpha=\beta=\gamma=\kappa=1$; see figures), with the corresponding ones in the natural 

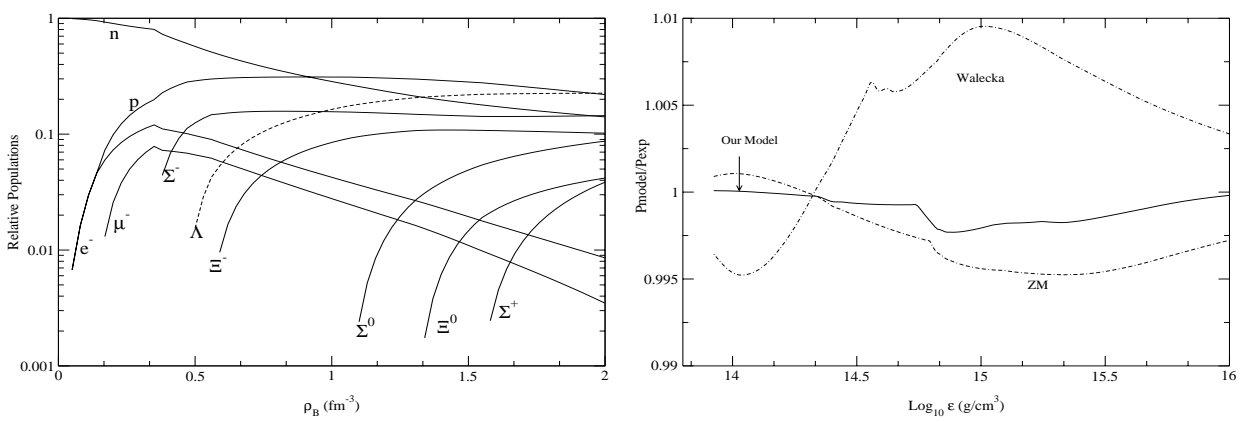

FIGURE 1. Left panel: relative populations. Right panel: EoS of nuclear matter. Compared are the results of our model (Eq. 3 with $\alpha=\beta=\gamma=\kappa=1$ ), Walecka[1], ZM[5] and exponential (Eq. 4) models.

limit, considering for simplicity only the scalar sector of our approach:

$$
\left.g_{\alpha \lambda B}^{\star}\right|_{\alpha \rightarrow \infty} \rightarrow \exp \left(-\frac{g_{\sigma} \sigma+g_{\delta} \tau_{3 B} \delta_{3}}{M_{B}}\right) g_{\lambda B} ; \lambda=\sigma, \omega, \rho, \delta
$$

\section{RESULTS AND CONCLUSIONS}

For few-body physics, the naive dimensional analysis and naturalness play a major role in effective field theory[5] bringing high precision to the theory. It is desirable to extend such precision to many-body physics and there are attempts to realize such a program. Here we show that NDA and naturalness, in combination with experiment, could represent a relevant criterium to select a model among others in the description of global static properties of neutron stars.

\section{REFERENCES}

1. $\quad$ B. D. Serot and J. D. Walecka, Int. J. Mod. Phys. E6 (1997) 515.

2. G.E. Brown and M. Rho, Phys. Rev. Lett. 66 (1991) 2720.

3. G.E. Brown and M. Rho, Nucl. Phys. A596 (1996) 503.

4. U. van Kolck, L. J. Abu-Raddad and D. M. Cardamone, in New States of Matter in Hadronic Interactions, PASI 2002, AIP Conf. Proceedings 631 (2002) 191.

5. M. Razeira et al. (contribution to this volume). 
Copyright of AIP Conference Proceedings is the property of American Institute of Physics and its content may not be copied or emailed to multiple sites or posted to a listserv without the copyright holder's express written permission. However, users may print, download, or email articles for individual use. 